TAIWANESE JOURNAL OF MATHEMATICS

Vol. 10, No. 2, pp. 441-465, February 2006

This paper is available online at http://www.math.nthu.edu.tw/tjm/

\title{
QUANTITATIVE EQUI-UNIFORM APPROXIMATION PROCESSES OF INTEGRAL OPERATORS IN BANACH SPACES
}

\author{
Toshihiko Nishishiraho
}

\begin{abstract}
We give quantitative estimates of the rate of convergence of equiuniform approximation processes of integral operators in Banach spaces in terms of the modulus of continuity of functions to be approximated and higher order absolute moments of approximate kernels. Furthermore, applications are presented for various equi-uniform summation processes, interpolation type operators, convolution type operators, and several concrete examples of approximating operators are also provided.
\end{abstract}

\section{INTRODUCTION}

Let $(E,\|\cdot\|)$ be a Banach space and let $(X, d)$ be a metric space. Let $B(X, E)$ denote the Banach space of all $E$-valued bounded functions on $X$ with the supremum norm. $B C(X, E)$ stands for the closed linear subspace of $B(X, E)$ consisting of all $E$-valued bounded continuous functions on $X$. Also, we denote by $C(X, E)$ the linear space consisting of all $E$-valued continuous functions on $X$. Let $X_{0}$ be a subset of $X$. Let $\mathfrak{K}=\left\{K_{\alpha, \lambda}: \alpha \in D, \lambda \in \Lambda\right\}$ be a family of operators from $B C(X, E)$ to $B\left(X_{0}, E\right)$, where $D$ is a directed set and $\Lambda$ is an index set. Then $\mathfrak{K}$ is called an equi-uniform approximation process on $B C(X, E)$ if for all $F \in B C(X, E)$,

$$
\lim _{\alpha}\left\|K_{\alpha, \lambda}(F)(x)-F(x)\right\|=0 \quad \text { uniformly in } \lambda \in \Lambda, x \in X_{0} .
$$

We here consider a family $\mathfrak{K}$ of integral operators on $B C(X, E)$ defined as follows:

Received August 17, 2004; Revised November 19, 2004.

2000 Mathematics Subject Classification: 41A35, 41A25.

Key words and phrases: Approximation process, Bochner integral, Integral operator, Modulus of continuity, Majorant, Absolute moment, Summation process, Interpolation type operator, Convolution type operator.

Dedicated to Professor Hang-Chin Lai on his seventieth birthday. 
Let $Y$ be a separable topological space and let $\mu$ be a Borel measure on $Y$. Let $\left\{\xi_{\alpha, \lambda}: \alpha \in D, \lambda \in \Lambda\right\}$ be a family of continuous mappings from $Y$ to $X$ and let $\mathfrak{A}=\left\{\chi_{\alpha, \lambda}(x ; \cdot): \alpha \in D, \lambda \in \Lambda, x \in X\right\}$ be a family of functions in $L^{1}(Y, \mu)$, which denotes the Banach space of all $\mu$-integrable functions $\chi$ on $Y$ with the norm

$$
\|\chi\|_{1}=\int_{Y}|\chi(y)| d \mu(y) .
$$

Then we define an integral operator by the form

$$
K_{\alpha, \lambda}(F)(x)=\int_{Y} \chi_{\alpha, \lambda}(x ; y) F\left(\xi_{\alpha, \lambda}(y)\right) d \mu(y) \quad(F \in B C(X, E)),
$$

which exists as a Bochner integral. In [12] we studied the convergence of equiuniform approximation processes of integral operators defined by (1).

The purpose of this paper is to give quantitative estimates of the rate of its convergence in terms of the modulus of continuity of functions to be approximated and higher order absolute moments of approximate kernels. Consequently, the results obtained in this paper are refinements of the estimates of the degree of approximation given in [13]. Furthermore, applications are presented for various summation processes, interpolation type operators, convolution type operators, and several concrete examples of approximating operators are also provided.

\section{Auxiliary Results}

Let $(Z, \rho)$ be a metric space. Let $F$ be a bounded mapping from $X$ to $Z$. Then we define

$\omega(F, \delta)=\omega(X, Z ; F, \delta)=\sup \{\rho(F(x), F(t)): x, t \in X, d(x, t) \leq \delta\} \quad(\delta \geq 0)$,

which is called the modulus of continuity of $F$.

Lemma 2.1. If $\omega$ is a nonnegative, monotone increasing subadditive function on $[0, \infty)$, then $\omega(\xi \delta) \leq(1+\xi) \omega(\delta)$ for all $\xi, \delta \geq 0$.

Proof. It follows from induction on $n$ that $\omega(n \delta) \leq n \omega(\delta)$ for all $n \in \mathbb{N}$ and all $\delta \geq 0$. Therefore, if $\xi \geq 1$, then denoting the largest positive integer not exceeding $\xi$ by $m$, we have $\omega(\xi \delta) \leq \omega((m+1) \delta) \leq(m+1) \omega(\delta) \leq(\xi+1) \omega(\delta)$. If $0 \leq \xi<1$, then $\omega(\xi \delta) \leq \omega(\delta) \leq(1+\xi) \omega(\delta)$.

$(X, d)$ is said to be quasi-convex if $x, y \in X, d(x, y) \leq a+b, a, b \geq 0,(a, b) \neq$ $(0,0)$, then there exists a point $z \in X$ such that $d(x, z) \leq a$ and $d(z, y) \leq b$. Let $(T, \tau)$ be a metric linear space. If $\tau(x, y)=\tau(x+z, y+z)$ for all $x, y, z \in T$, 
then $\tau$ is called a translation invariant metric function. A real-valued function $\varphi$ on a linear space $V$ is said to be starshaped if $\varphi(\beta x) \leq \beta \varphi(x)$ for all $x \in V$ and all $\beta \in[0,1]$.

Lemma 2.2. Let $(T, \tau)$ be a translation invariant metric linear space such that $\tau(\cdot, 0)$ is starshaped on $T$. If $W$ is a convex subset of $T$, then $(W, \tau)$ is quasi-convex.

Proof. Let $x, y \in W, \tau(x, y) \leq a+b, a, b \geq 0,(a, b) \neq(0,0)$. Then we take $z=(b /(a+b)) x+(a /(a+b)) y$, which belongs to $W$. Now, we have

$\tau(x, z)=\tau\left(x-\frac{b}{a+b} x, \frac{a}{a+b} y\right)=\tau\left(\frac{a}{a+b}(x-y), 0\right) \leq \frac{a}{a+b} \tau(x-y, 0) \leq a$ and similarly, the inequality $\tau(z, y) \leq b$ holds.

Lemma 2.3. If $(X, d)$ is quasi-convex, then $\omega(F, \cdot)$ is subadditive on $[0, \infty)$.

Proof. Suppose that $x, t \in X, d(x, t) \leq \delta_{1}+\delta_{2}, \delta_{1}, \delta_{2} \geq 0,\left(\delta_{1}, \delta_{2}\right) \neq(0,0)$ and $x \neq t$. We put $\xi=d(x, t) /\left(\delta_{1}+\delta_{2}\right)$. Then by the quasi-convexity of $d$, there exists a point $z \in X$ such that $d(x, z) \leq \xi \delta_{1} \leq \delta_{1}$ and $d(z, t) \leq \xi \delta_{2} \leq \delta_{2}$. Therefore, we have

$$
\rho(F(x), F(t)) \leq \rho(F(x), F(z))+\rho(F(z), F(t)) \leq \omega\left(F, \delta_{1}\right)+\omega\left(F, \delta_{2}\right),
$$

which always holds for $x=t$. Thus we have $\omega\left(F, \delta_{1}+\delta_{2}\right) \leq \omega\left(F, \delta_{1}\right)+\omega\left(F, \delta_{2}\right)$, which holds also for $\delta_{1}=\delta_{2}=0$.

Now, we consider the following condition: There exist constants $C \geq 1$ and $K>0$ such that

$$
\omega(F, \xi \delta) \leq(C+K \xi) \omega(F, \delta)
$$

for every bounded mapping $F$ from $X$ to $Z$ and for every $\xi, \delta \geq 0$.

The following lemma gives sufficient condition such that (2) holds with $C=$ $K=1$, which can be more convenient for later applications and generalizes [10, Lemma 3]:

\section{Lemma 2.4.}

(a) If $(X, d)$ is quasi-convex, then (2) holds with $C=K=1$.

(b) If $X$ is a convex subset of a metric linear space with the translation invariant metric function d and if $d(\cdot, 0)$ is starshaped, then (2) holds with $C=K=1$. In particular, if $X$ is a convex subset of a normed linear space, then (2) holds with $C=K=1$. 
Proof. Let $\omega(\cdot)=\omega(F, \cdot)$. Then Part (a) follows from Lemmas 2.1 and 2.3. Also, Part (b) follows from Lemma 2.2 and Part (a).

Let $V(X, E)$ denote the linear space of all $E$-valued functions on $X$. For any scalar-valued function $v$ on $X$ and $a \in E$, we define $(v \otimes a)(x)=v(x) a$ for all $x \in X .1_{X}$ denotes the unit function defined by $1_{X}(x)=1$ for all $x \in X$. Let $A(X, E)$ be a linear subspace of $V(X, E)$ and let $\varphi$ be a mapping from $A(X, E)$ to $E$. A positive linear functional $\nu$ on $A(X, \mathbb{R})$ is called a majorant (or dominant) functional of $\varphi$ if $\|\varphi(F)\| \leq \nu(v)$ whenever $F \in A(X, E), v \in A(X, \mathbb{R})$ satisfy

$$
\|F(t)\| \leq v(t) \quad \text { for all } t \in X .
$$

Let $L$ be a mapping from $A(X, E)$ to $V\left(X_{0}, E\right)$. A positive linear operator $S$ from $A(X, \mathbb{R})$ to $V\left(X_{0}, \mathbb{R}\right)$ is called a majorant (or dominant ) operator of $L$ if (3) implies that $\|L(F)(x)\| \leq S(v)(x)$ for all $x \in X_{0}$.

From now on, in order to achieve our purpose we suppose that $Z=E, \rho(x, t)=$ $\|x-t\|(x, t \in Z)$ and that (2) holds.

Lemma 2.5. Let $\varphi$ be a mapping from $A(X, E)$ to $E$ having a majorant functional $\nu$. Let $p \geq 1$ and $x \in X$. Suppose that

$$
\left\{1_{X} \otimes a: a \in E\right\} \subseteq A(X, E), \quad\left\{1_{X}, d(x, \cdot), d^{p}(x, \cdot)\right\} \subseteq A(X, \mathbb{R}) .
$$

Then for all $F \in A(X, E)$ and all $\delta>0$,

$$
\left\|\varphi\left(F-1_{X} \otimes F(x)\right)\right\| \leq\left(C \nu\left(1_{X}\right)+K m_{\nu}(x ; p, \delta)\right) \omega(F, \delta),
$$

where

$$
m_{\nu}(x ; p, \delta)=\min \left\{\delta^{-p} \nu\left(d^{p}(x, \cdot)\right), \delta^{-1} \nu\left(1_{X}\right)^{1-1 / p} \nu\left(d^{p}(x, \cdot)\right)^{1 / p}\right\} .
$$

Proof. Let $t \in X$. If $d(x, t)>\delta$, then by (2) we have

$$
\|F(t)-F(x)\| \leq\left(C+K \delta^{-p} d^{p}(x, t)\right) \omega(F, \delta),
$$

which always holds for $d(x, t) \leq \delta$ on account of $C \geq 1$. Therefore, we have

$$
\left\|\varphi\left(F-1_{X} \otimes F(x)\right)\right\| \leq\left(C \nu\left(1_{X}\right)+K \delta^{-p} \nu\left(d^{p}(x, \cdot)\right)\right) \omega(F, \delta) .
$$

On the other hand, since

$$
\|F(t)-F(x)\| \leq\left(C+K \delta^{-1} d(x, t)\right) \omega(F, \delta),
$$

using Hölder's inequality we get

$$
\left\|\varphi\left(F-1_{X} \otimes F(x)\right)\right\| \leq\left(C \nu\left(1_{X}\right)+K \delta^{-1} \nu\left(d^{p}(x, \cdot)\right)^{1 / p} \nu\left(1_{X}\right)^{1-1 / p}\right) \omega(F, \delta),
$$


which together with (5) establishes (4) for $p>1$. If $p=1$, then (5) is clearly identical with (4).

Lemma 2.6. Let $L$ be a mapping from $A(X, E)$ to $V\left(X_{0}, E\right)$ having a majorant operator $S$ and let $p \geq 1$. Suppose that

$\left\{1_{X} \otimes a: a \in E\right\} \subseteq A(X, E), \quad\left\{1_{X}\right\} \cup\left\{d(x, \cdot), d^{p}(x, \cdot): x \in X_{0}\right\} \subseteq A(X, \mathbb{R})$.

Then for all $F \in A(X, E), x \in X_{0}$ and all $\delta>0$,

$$
\left\|L\left(F-1_{X} \otimes F(x)\right)(x)\right\| \leq\left(C S\left(1_{X}\right)(x)+K m_{S}(x ; p, \delta)\right) \omega(F, \delta),
$$

where

$$
m_{S}(x ; p, \delta)=\min \left\{\delta^{-p} S\left(d^{p}(x, \cdot)\right)(x), \delta^{-1}\left(S\left(1_{X}\right)(x)\right)^{1-1 / p}\left(S\left(d^{p}(x, \cdot)\right)(x)\right)^{1 / p}\right\} .
$$

Proof. Let $x \in X_{0}$ be fixed. Then we apply Lemma 2.5 to $\varphi(\cdot)=L(\cdot)(x)$ and $\nu(\cdot)=S(\cdot)(x)$.

Note that Lemma 2.6 generalizes [4, Theorem 2.1] and improves the estimate by means of higher order absolute moments.

Lemma 2.7. Let $\{\chi(x ; \cdot): x \in X\}$ be a family of functions in $L^{1}(Y, \mu), \tau$ a continuous mapping from $Y$ to $X$ and $p \geq 1$. Assume that $\chi(x ; \cdot) d^{p}(x, \tau(\cdot)) \in$ $L^{1}(Y, \mu)$ for each $x \in X_{0}$. Then for all $F \in B C(X, E), x \in X_{0}$ and all $\delta>0$,

$$
\left\|\int_{Y} \chi(x ; y)(F(\tau(y))-F(x)) d \mu(y)\right\| \leq\left(C\|\chi(x ; \cdot)\|_{1}+K c(x ; p, \delta)\right) \omega(F, \delta),
$$

where

$c(x ; p, \delta)=\min \left\{\delta^{-p}\left\|\chi(x ; \cdot) d^{p}(x, \tau(\cdot))\right\|_{1}, \delta^{-1}\|\chi(x ; \cdot)\|_{1}^{1-1 / p}\left\|\chi(x ; \cdot) d^{p}(x, \tau(\cdot))\right\|_{1}^{1 / p}\right\}$.

Proof. Let $x \in X_{0}$ be fixed. Let

$$
A(X, E):=\left\{F \in C(X, E): \chi(x ; \cdot)\|F(\tau(\cdot))\| \in L^{1}(Y, \mu)\right\}
$$

and

$$
A(X, \mathbb{R}):=\left\{u \in C(X, \mathbb{R}): \chi(x ; \cdot) u(\tau(\cdot)) \in L^{1}(Y, \mu)\right\} .
$$

Then by the hypotheses, $1_{X}$ and $d^{p}(x, \cdot)$ belong to $A(X, \mathbb{R})$, and so Hölder's inequality implies $d(x, \cdot) \in A(X, \mathbb{R})$. Now, we define

$$
\varphi(F)=\int_{Y} \chi(x ; y) F(\tau(y)) d \mu(y) \quad(F \in A(X, E))
$$


and

$$
\nu(u)=\int_{Y}|\chi(x ; y)| u(\tau(y)) d \mu(y) \quad(u \in A(X, \mathbb{R})) .
$$

Then $\nu$ is a majorant functional of $\varphi$, and so applying Lemma 2.5 to $F \in B C(X, E)$ $(\subset A(X, E))$ we obtain the desired result.

\section{Degree of Equi-uniform Approximation}

Let $p \geq 1$ be any fixed real number. We suppose that

$$
\chi_{\alpha, \lambda}(x ; \cdot) d^{p}\left(x, \xi_{\alpha, \lambda}(\cdot)\right) \in L^{1}(Y, \mu)
$$

for each $\alpha \in D, \lambda \in \Lambda$ and for each $x \in X_{0}$ and define

$$
\mu_{\alpha, \lambda}(x ; p)=\left\|\chi_{\alpha, \lambda}(x ; \cdot) d^{p}\left(x, \xi_{\alpha, \lambda}(\cdot)\right)\right\|_{1},
$$

which is called the $p$ th absolute moment of $\chi_{\alpha, \lambda}(x ; \cdot)$. Furthermore, we asuume that for each $\alpha \in D$,

$$
c_{\alpha}:=\sup \left\{\left\|\chi_{\alpha, \lambda}(x ; \cdot)\right\|_{1}: \lambda \in \Lambda, x \in X_{0}\right\}<\infty
$$

and

$$
0<\mu_{\alpha}(p):=\left(\sup \left\{\mu_{\alpha, \lambda}(x ; p): \lambda \in \Lambda, x \in X_{0}\right\}\right)^{1 / p}<\infty .
$$

For any $\alpha \in D, F \in B C(X, E)$ we define

$$
\begin{aligned}
& \gamma_{\alpha}=\sup \left\{\left|\int_{Y} \chi_{\alpha, \lambda}(x ; y) d \mu(y)-1\right|: \lambda \in \Lambda, x \in X_{0}\right\}, \\
& E_{\alpha}(F)=\sup \left\{\left\|K_{\alpha, \lambda}(F)(x)-F(x)\right\|: \lambda \in \Lambda, x \in X_{0}\right\}
\end{aligned}
$$

and

$$
\|F\|_{S}=\sup \{\|F(x)\|: x \in S\} \quad(S \subseteq X) .
$$

Obviously, $\mathfrak{K}$ is an equi-uniform approximation process on $B C(X, E)$ if and only if $\lim _{\alpha} E_{\alpha}(F)=0$ for every $F \in B C(X, E)$.

If $\chi_{\alpha, \lambda}(x ; y) \geq 0(\mu$-a.e. $y \in Y)$ for all $\alpha \in D, \lambda \in \Lambda$ and all $x \in X_{0}$, then $\mathfrak{A}$ is said to be positive. If

$$
\int_{Y} \chi_{\alpha, \lambda}(x ; y) d \mu(y)=1
$$

for all $\alpha \in D, \lambda \in \Lambda$ and all $x \in X_{0}$, then $\mathfrak{A}$ is said to be normal.

From now on, let $\left\{\epsilon_{\alpha}\right\}_{\alpha \in D}$ be a net of positive real numbers. 
Theorem 3.1. For all $F \in B C(X, E)$ and all $\alpha \in D$,

$$
E_{\alpha}(F) \leq\|F\|_{X_{0}} \gamma_{\alpha}+\gamma_{\alpha}(p) \omega\left(F, \epsilon_{\alpha} \mu_{\alpha}(p)\right)
$$

where

$\gamma_{\alpha}(p)=\sup \left\{C\left\|\chi_{\alpha, \lambda}(x ; \cdot)\right\|_{1}+K \min \left\{\epsilon_{\alpha}^{-1}\left\|\chi_{\alpha, \lambda}(x ; \cdot)\right\|_{1}^{1-1 / p}, \epsilon_{\alpha}^{-p}\right\}: \lambda \in \Lambda, x \in X_{0}\right\}$.

In particular, if $\mathfrak{A}$ is positive and normal, then

$$
E_{\alpha}(F) \leq\left(C+K \min \left\{\epsilon_{\alpha}^{-1}, \epsilon_{\alpha}^{-p}\right\}\right) \omega\left(F, \epsilon_{\alpha} \mu_{\alpha}(p)\right) .
$$

Proof. Let $F \in B C(X, E)$ and $x \in X_{0}$. Then for all $\alpha \in D, \lambda \in \Lambda$ we have

$$
\begin{gathered}
\left\|K_{\alpha, \lambda}(F)(x)-F(x)\right\| \leq\left|\int_{Y} \chi_{\alpha, \lambda}(x ; y) d \mu(y)-1\right|\|F(x)\| \\
+\left\|\int_{Y} \chi_{\alpha, \lambda}(x ; y)\left(F\left(\xi_{\alpha, \lambda}(y)\right)-F(x)\right) d \mu(y)\right\|=J_{\alpha, \lambda}^{(1)}(x)+J_{\alpha, \lambda}^{(2)}(x),
\end{gathered}
$$

say. Obviously, we have $J_{\alpha, \lambda}^{(1)}(x) \leq \gamma_{\alpha}\|F\|_{X_{0}}$. Now, applying Lemma 2.7 to $\chi(x ; \cdot)=\chi_{\alpha, \lambda}(x ; \cdot)$ and $\tau=\xi_{\alpha, \lambda}$, we obtain

$$
J_{\alpha, \lambda}^{(2)}(x) \leq\left(C\left\|\chi_{\alpha, \lambda}(x ; \cdot)\right\|_{1}+K c_{\alpha, \lambda}(x ; p, \delta)\right) \omega(F, \delta) \quad(\delta>0),
$$

where

$$
\begin{aligned}
& c_{\alpha, \lambda}(x ; p, \delta)=\min \left\{\delta^{-p} \mu_{\alpha, \lambda}(x ; p), \delta^{-1}\left\|\chi_{\alpha, \lambda}(x ; \cdot)\right\|_{1}^{1-1 / p} \mu_{\alpha, \lambda}(x ; p)^{1 / p}\right\} \\
& \leq \min \left\{\delta^{-p} \mu_{\alpha}(p)^{p}, \delta^{-1}\left\|\chi_{\alpha, \lambda}(x ; \cdot)\right\|_{1}^{1-1 / p} \mu_{\alpha}(p)\right\} .
\end{aligned}
$$

Putting $\delta=\epsilon_{\alpha} \mu_{\alpha}(p)$ in the above inequality and taking the supremum over all $\lambda \in \Lambda$ and all $x \in X_{0}$, (7) establishes the desired estimate (6).

Let $\Phi$ be a nonnegative real-valued function on $X_{0} \times X$ and let $s>0$. If

$$
\chi_{\alpha, \lambda}(x ; \cdot) \Phi^{s}\left(x, \xi_{\alpha, \lambda}(\cdot)\right) \in L^{1}(Y, \mu) \quad\left(\alpha \in D, \lambda \in \Lambda, x \in X_{0}\right),
$$

then $\mu_{\alpha, \lambda}(\Phi ; x ; s):=\left\|\chi_{\alpha, \lambda}(x ; \cdot) \Phi^{s}\left(x, \xi_{\alpha, \lambda}(\cdot)\right)\right\|_{1}$ is called the $s$ th absolute moment of $\chi_{\alpha, \lambda}(x ; \cdot)$ with respect to $\Phi$.

Suppose that there exist positive constants $q$ and $\kappa$ such that

$$
d^{q}(x, t) \leq \kappa \Phi(x, t) \quad \text { for all }(x, t) \in X_{0} \times X
$$

and that

$$
0<\mu_{\alpha}(\Phi ; p / q):=\left(\sup \left\{\mu_{\alpha, \lambda}(\Phi ; x ; p / q): \lambda \in \Lambda, x \in X_{0}\right\}\right)^{1 / p}<\infty
$$


is satisfied.

Theorem 3.2. For all $F \in B C(X, E)$ and all $\alpha \in D$,

$$
E_{\alpha}(F) \leq\|F\|_{X_{0}} \gamma_{\alpha}+\gamma_{\alpha}(p, q) \omega\left(F, \epsilon_{\alpha} \mu_{\alpha}(\Phi ; p / q)\right),
$$

where

$$
\begin{aligned}
& \gamma_{\alpha}(p, q) \\
& =\sup \left\{C\left\|\chi_{\alpha, \lambda}(x ; \cdot)\right\|_{1}+K \min \left\{\kappa^{1 / q} \epsilon_{\alpha}^{-1}\left\|\chi_{\alpha, \lambda}(x ; \cdot)\right\|_{1}^{1-1 / p}, \kappa^{p / q} \epsilon_{\alpha}^{-p}\right\}: \lambda \in \Lambda, x \in X_{0}\right\} .
\end{aligned}
$$

In particular, if $\mathfrak{A}$ is positive and normal, then

$$
E_{\alpha}(F) \leq\left(C+K \min \left\{\kappa^{1 / q} \epsilon_{\alpha}^{-1}, \kappa^{p / q} \epsilon_{\alpha}^{-p}\right\}\right) \omega\left(F, \epsilon_{\alpha} \mu_{\alpha}(\Phi ; p / q)\right) .
$$

Proof. By (8), we have

$$
\mu_{\alpha, \lambda}(x ; p) \leq \kappa^{p / q} \mu_{\alpha, \lambda}(\Phi ; x ; p / q) \quad\left(\alpha \in D, \lambda \in \Lambda, x \in X_{0}\right),
$$

which implies $\mu_{\alpha}(p) \leq \kappa^{1 / q} \mu_{\alpha}(\Phi ; p / q)$. Therefore, by putting $\kappa^{-1 / q} \epsilon_{\alpha}$ instead of $\epsilon_{\alpha}$, the desired estimate (9) follows from (6).

In the rest of this section, we restrict the integral operators $K_{\alpha, \lambda}$ defined by (1) to the subclass of $B C(X, E)$ as follows:

Let $E_{0}$ be a subset of $E$ and $\mathfrak{T}=\{T(x): x \in X\}$ a family of mappings from $E_{0}$ to $E$ such that for each $f \in E_{0}$, the mapping $x \mapsto T(x)(f)$ is strongly continuous and bounded on $X$ and let $L_{\alpha, \lambda}$ denote the restriction of $K_{\alpha, \lambda}$ to $\left\{T(\cdot)(f): f \in E_{0}\right\}$, i.e.,

$$
L_{\alpha, \lambda}(x)(f)=\int_{Y} \chi_{\alpha, \lambda}(x ; y) T\left(\xi_{\alpha, \lambda}(y)\right)(f) d \mu(y) \quad\left(f \in E_{0}\right) .
$$

Shaw [15] considered the special case of (10) in the setting of certain spaces of operator-valued functions and obtained several representation formulas for strongly continuous semigroups of bounded linear operators on Banach spaces.

The family $\mathfrak{L}=\left\{L_{\alpha, \lambda}(x): \alpha \in D, \lambda \in \Lambda, x \in X\right\}$ is called an equi-uniform T-approximation process on $E_{0}$ if for every $f \in E_{0}$,

$$
\lim _{\alpha}\left\|L_{\alpha, \lambda}(x)(f)-T(x)(f)\right\|=0 \quad \text { uniformly in } \lambda \in \Lambda, x \in X_{0} .
$$

Concerning the rate of convergence behavior of (11), we define

$$
\omega_{\mathfrak{T}}(f, \delta)=\sup \{\|T(x)(f)-T(t)(f)\|: x, t \in X, d(x, t) \leq \delta\} \quad\left(f \in E_{0}, \delta \geq 0\right),
$$


which is called the modulus of continuity of $f$ associated with $\mathfrak{T}$, and

$$
e_{\alpha}(f)=\sup \left\{\left\|L_{\alpha, \lambda}(x)(f)-T(x)(f)\right\|: \lambda \in \Lambda, x \in X_{0}\right\} .
$$

Now, since

$$
\omega_{\mathfrak{T}}(f, \delta)=\omega(T(\cdot)(f), \delta), \quad e_{\alpha}(f)=E_{\alpha}(T(\cdot)(f)) \quad\left(f \in E_{0}, \delta \geq 0, \alpha \in D\right),
$$

Theorems 3.1 and 3.2 establish the following results which estimate the rate of convergence of the equi-uniform $\mathfrak{T}$-approximation process $\mathfrak{L}$ on $E_{0}$ :

Corollary 3.3. For all $f \in E_{0}$ and all $\alpha \in D$,

$$
e_{\alpha}(f) \leq\|T(\cdot)(f)\|_{X_{0}} \gamma_{\alpha}+\gamma_{\alpha}(p) \omega_{\mathfrak{T}}\left(f, \epsilon_{\alpha} \mu_{\alpha}(p)\right) .
$$

In particular, if $\mathfrak{A}$ is positive and normal, then

$$
e_{\alpha}(f) \leq\left(C+K \min \left\{\epsilon_{\alpha}^{-1}, \epsilon_{\alpha}^{-p}\right\}\right) \omega_{\mathfrak{T}}\left(f, \epsilon_{\alpha} \mu_{\alpha}(p)\right) .
$$

Corollary 3.4. For all $f \in E_{0}$ and all $\alpha \in D$,

$$
e_{\alpha}(f) \leq\|T(\cdot)(f)\|_{X_{0}} \gamma_{\alpha}+\gamma_{\alpha}(p, q) \omega_{\mathfrak{T}}\left(f, \epsilon_{\alpha} \mu(\Phi ; p / q)\right) .
$$

In particular, if $\mathfrak{A}$ is positive and normal, then

$$
e_{\alpha}(f) \leq\left(C+K \min \left\{\kappa^{1 / q} \epsilon_{\alpha}^{-1}, \kappa^{p / q} \epsilon_{\alpha}^{-p}\right\}\right) \omega_{\mathfrak{T}}\left(f, \epsilon_{\alpha} \mu_{\alpha}(\Phi ; p / q)\right) .
$$

\section{Equi-uniform Summation Processes}

Let $\mathcal{A}=\left\{a_{\alpha, m}^{(\lambda)}: \alpha \in D, m \in \mathbb{N}_{0}, \lambda \in \Lambda\right\}$ be a family of scalars. $\mathcal{A}$ is said to be regular if it satisfies the following conditions:

$(A-1)$ For each $m \in \mathbb{N}_{0}, \lim _{\alpha} a_{\alpha, m}^{(\lambda)}=0 \quad$ uniformly in $\lambda \in \Lambda$.

$(A-2) \lim _{\alpha} \sum_{m=0}^{\infty} a_{\alpha, m}^{(\lambda)}=1 \quad$ uniformly in $\lambda \in \Lambda$.

$(A-3)$ For each $\alpha \in D, \lambda \in \Lambda, a_{\alpha}^{(\lambda)}:=\sum_{m=0}^{\infty}\left|a_{\alpha, m}^{(\lambda)}\right|<\infty$, and there exists $\alpha_{0} \in D$ such that $\sup \left\{a_{\alpha}^{(\lambda)}: \alpha \geq \alpha_{0}, \alpha \in D, \lambda \in \Lambda\right\}<\infty$.

$\mathcal{A}$ is said to be stochastic if

$$
a_{\alpha, m}^{(\lambda)} \geq 0 \quad\left(\alpha \in D, m \in \mathbb{N}_{0}, \lambda \in \Lambda\right) \quad \text { and } \quad \sum_{m=0}^{\infty} a_{\alpha, m}^{(\lambda)}=1 \quad(\alpha \in D, \lambda \in \Lambda) .
$$


Obviously, if $\mathcal{A}$ is stochastic, then Conditions (A-2) and (A-3) are automatically satisfied.

A sequence $\left\{f_{m}\right\}_{m \in \mathbb{N}_{0}}$ of elements in $E$ is said to be $\mathcal{A}$-summable to $f$ if

$$
\lim _{\alpha}\left\|\sum_{m=0}^{\infty} a_{\alpha, m}^{(\lambda)} f_{m}-f\right\|=0 \quad \text { uniformly in } \lambda \in \Lambda,
$$

where it is assumed that the series in (12) converges for each $\alpha \in D$ and $\lambda \in \Lambda$.

Concerning the relation between the regularity of $\mathcal{A}$ and $\mathcal{A}$-summability, $\mathcal{A}$ is regular if and only if every convergent sequence in $E$ is $\mathcal{A}$-summable to its limit (cf. [1, 9]). Also, for several important examples of generic entories $a_{\alpha, m}^{(\lambda)}$ of $\mathcal{A}$ with $D=\mathbb{N}_{0}$, see [9] (cf. $[1,5,7,14]$ ).

Now, let $\left\{\xi_{n}\right\}_{n \in \mathbb{N}_{0}}$ be a sequence of continuous mappings from $Y$ to $X$ and let $\left\{\chi_{n}(x ; \cdot): n \in \mathbb{N}_{0}, x \in X\right\}$ be a family of functions in $L^{1}(Y, \mu)$ such that

$$
b_{\alpha, \lambda}(x):=\sum_{m=0}^{\infty} \int_{Y}\left|a_{\alpha, m}^{(\lambda)} \chi_{m}(x ; y)\right| d \mu(y)<\infty \quad(\alpha \in D, \lambda \in \Lambda, x \in X) .
$$

We define

$$
K_{n}(F)(x)=\int_{Y} \chi_{n}(x ; y) F\left(\xi_{n}(y)\right) d \mu(y) \quad(F \in B C(X, E))
$$

and

$$
L_{n}(x)(f)=\int_{Y} \chi_{n}(x ; y) T\left(\xi_{n}(y)\right)(f) d \mu(y) \quad\left(f \in E_{0}\right) .
$$

The sequence $\left\{K_{n}\right\}_{n \in \mathbb{N}_{0}}$ is called an equi-uniform $\mathcal{A}$-summation process on $B C(X$, $E$ ) if the family $\mathfrak{K}=\left\{K_{\alpha, \lambda}: \alpha \in D, \lambda \in \Lambda\right\}$ is an equi-uniform approximation process on $B C(X, E)$, where each $K_{\alpha, \lambda}$ is defined by

$$
K_{\alpha, \lambda}(F)(x)=\sum_{m=0}^{\infty} a_{\alpha, m}^{(\lambda)} K_{m}(F)(x) \quad(F \in B C(X, E)) .
$$

The family $\left\{L_{n}(x): n \in \mathbb{N}_{0}, x \in X\right\}$ is called an equi-uniform $\mathfrak{T}$ - $\mathcal{A}$-summation process on $E_{0}$ if the family $\mathfrak{L}=\left\{L_{\alpha, \lambda}(x): \alpha \in D, \lambda \in \Lambda, x \in X\right\}$ is an equiuniform $\mathfrak{T}$-approximation process on $E_{0}$, where each $L_{\alpha, \lambda}(x)$ is defined by

$$
L_{\alpha, \lambda}(x)(f)=\sum_{m=0}^{\infty} a_{\alpha, m}^{(\lambda)} L_{m}(x)(f) \quad\left(f \in E_{0}\right) .
$$

Let $K_{\alpha, \lambda}$ and $L_{\alpha, \lambda}$ be defined by (15) and (16), respectively. Let $p \geq 1$ and suppose that $\chi_{n}(x ; \cdot) d^{p}\left(x, \xi_{n}(\cdot)\right) \in L^{1}(Y, \mu)$ for all $n \in \mathbb{N}_{0}$ and all $x \in X_{0}$. 
Theorem 4.1. Let $\left\{\epsilon_{\alpha}\right\}_{\alpha \in D}$ be a net of positive real numbers. Then for all $F \in B C(X, E)$ and all $\alpha \in D$,

$$
E_{\alpha}(F) \leq\|F\|_{X_{0}} \tau_{\alpha}+\tau_{\alpha}(p) \omega\left(F, \epsilon_{\alpha} \nu_{\alpha}(p)\right)
$$

where

$$
\begin{gathered}
\tau_{\alpha}=\sup \left\{\left|\sum_{m=0}^{\infty} a_{\alpha, m}^{(\lambda)} \int_{Y} \chi_{m}(x ; y) d \mu(y)-1\right|: \lambda \in \Lambda, x \in X_{0}\right\}, \\
\tau_{\alpha}(p)=\sup \left\{C b_{\alpha, \lambda}(x)+K \min \left\{\epsilon_{\alpha}^{-p}, \epsilon_{\alpha}^{-1} b_{\alpha, \lambda}(x)^{1-1 / p}\right\}: \lambda \in \Lambda, x \in X_{0}\right\}
\end{gathered}
$$

and

$$
\nu_{\alpha}(p)=\left(\sup \left\{\sum_{m=0}^{\infty}\left|a_{\alpha, m}^{(\lambda)}\right|\left\|\chi_{m}(x ; \cdot) d^{p}\left(x, \xi_{m}(\cdot)\right)\right\|_{1}: \lambda \in \Lambda, x \in X_{0}\right\}\right)^{1 / p} .
$$

Proof. For all $\lambda \in \Lambda$ and all $x \in X_{0}$, we have

$$
\begin{gathered}
\left\|K_{\alpha, \lambda}(F)(x)-F(x)\right\| \leq \sum_{m=0}^{\infty}\left|a_{\alpha, m}^{(\lambda)}\right||| \int_{Y} \chi_{m}(x ; y)\left(F\left(\xi_{m}(y)\right)-F(x)\right) d \mu(y) \| \\
+\left|\sum_{m=0}^{\infty} a_{\alpha, m}^{(\lambda)} \int_{Y} \chi_{m}(x ; y) d \mu(y)-1\right|\|F(x)\|=I_{\alpha, \lambda}^{(1)}(x)+I_{\alpha, \lambda}^{(2)}(x),
\end{gathered}
$$

say. Obviously, we have $I_{\alpha, \lambda}^{(2)}(x) \leq \tau_{\alpha}\|F\|_{X_{0}}$. Taking $\chi(x ; \cdot)=\chi_{m}(x ; \cdot)$ and $\tau=\xi_{m}$ in Lemma 2.7, we get

$$
I_{\alpha, \lambda}^{(1)}(x) \leq\left(C b_{\alpha, \lambda}(x)+K \sum_{m=0}^{\infty}\left|a_{\alpha, m}^{(\lambda)}\right| c_{m}(x ; p, \delta)\right) \omega(F, \delta) \quad(\delta>0),
$$

where

$$
\begin{aligned}
& c_{m}(x ; p, \delta) \\
& =\min \left\{\delta^{-p}\left\|\chi_{m}(x ; \cdot) d^{p}\left(x, \xi_{m}(\cdot)\right)\right\|_{1}, \delta^{-1}\left\|\chi_{m}(x ; \cdot)\right\|_{1}^{1-1 / p}\left\|\chi_{m}(x ; \cdot) d^{p}\left(x, \xi_{m}(\cdot)\right)\right\|_{1}^{1 / p}\right\} .
\end{aligned}
$$

Now, if $p>1$, then by Hölder's inequality we have

$$
\begin{aligned}
& \sum_{m=0}^{\infty}\left|a_{\alpha, m}^{(\lambda)}\right|\left\|\chi_{m}(x ; \cdot)\right\|_{1}^{1-1 / p}\left\|\chi_{m}(x ; \cdot) d^{p}\left(x ; \xi_{m}(\cdot)\right)\right\|_{1}^{1 / p} \\
& \quad \leq\left(\sum_{m=0}^{\infty}\left|a_{\alpha, m}^{(\lambda)}\right|\left\|\chi_{m}(x ; \cdot)\right\|_{1}\right)^{1-1 / p}\left(\sum_{m=0}^{\infty}\left|a_{\alpha, m}^{(\lambda)}\right|\left\|\chi_{m}(x ; \cdot) d^{p}\left(x, \xi_{m}(\cdot)\right)\right\|_{1}\right)^{1 / p}
\end{aligned}
$$


which clearly holds for $p=1$. Therefore, we have

$$
\sum_{m=0}^{\infty}\left|a_{\alpha, m}^{(\lambda)}\right| c_{m}(x ; p, \delta) \leq \min \left\{\delta^{-p} \nu_{\alpha}(p)^{p}, \delta^{-1} b_{\alpha, \lambda}(x)^{1-1 / p} \nu_{\alpha}(p)\right\},
$$

which together with (19) implies

$$
I_{\alpha, \lambda}^{(1)}(x) \leq\left(C b_{\alpha, \lambda}(x)+K \min \left\{\delta^{-p} \nu_{\alpha}(p)^{p}, \delta^{-1} b_{\alpha, \lambda}(x)^{1-1 / p} \nu_{\alpha}(p)\right\}\right) \omega(F, \delta) .
$$

Putting $\delta=\epsilon_{\alpha} \nu_{\alpha}(p)$ in the above inequality and taking the supremum over all $\lambda \in \Lambda$ and all $x \in X_{0}$ in (18), we obtain the desired estimate (17).

Corollary 4.2. Let $\left\{\epsilon_{\alpha}\right\}_{\alpha \in D}, \tau_{\alpha}, \tau_{\alpha}(p)$ and $\nu_{\alpha}(p)$ be as in Theorem 4.1. Then for all $f \in E_{0}$ and all $\alpha \in D$,

$$
e_{\alpha}(f) \leq\|T(\cdot)(f)\|_{X_{0}} \tau_{\alpha}+\tau_{\alpha}(p) \omega_{\mathfrak{T}}\left(f, \epsilon_{\alpha} \nu_{\alpha}(p)\right) .
$$

\section{INTERPOLATION TyPe OPERATORS}

Let $Y$ be a finite set. Then the integral operators given by (1) and (10) reduce to the interpolation type operators

$$
K_{\alpha, \lambda}(F)(x)=\sum_{y \in Y} \chi_{\alpha, \lambda}(x ; y) F\left(\xi_{\alpha, \lambda}(y)\right) \quad(F \in B C(X, E))
$$

and

$$
L_{\alpha, \lambda}(x)(f)=\sum_{y \in Y} \chi_{\alpha, \lambda}(x ; y) T\left(\xi_{\alpha, \lambda}(y)\right)(f) \quad\left(f \in E_{0}\right),
$$

respectively. Here we restrict ourselves to the following situation:

Let $1 \leq s \leq \infty$ be fixed and let $X$ be a convex subset of the $r$-dimensional Euclidean space $\mathbb{R}^{r}$ with the metric

$$
d(x, t)=d_{s}(x, t):= \begin{cases}\left(\sum_{i=1}^{r}\left|x_{i}-t_{i}\right|^{s}\right)^{1 / s} & (1 \leq s<\infty) \\ \max \left\{\left|x_{i}-t_{i}\right|: 1 \leq i \leq r\right\} & (s=\infty),\end{cases}
$$

where $x=\left(x_{1}, x_{2}, \ldots, x_{r}\right), t=\left(t_{1}, t_{2}, \ldots, t_{r}\right) \in \mathbb{R}^{r}$. Therefore, by Lemma 2.4 (b), (2) holds with $C=K=1$. For $i=1,2, \ldots, r, p_{i}$ denotes the $i$ th coordinate function on $\mathbb{R}^{r}$ defined by $p_{i}(x)=x_{i}$ for all $x=\left(x_{1}, x_{2}, \ldots, x_{r}\right) \in \mathbb{R}^{r}$. Then we have

$$
d_{s}^{q}(x, t) \leq c(q, r, s) \sum_{i=1}^{r}\left|p_{i}(x)-p_{i}(t)\right|^{q} \quad\left(x, t \in \mathbb{R}^{r}, q>0\right),
$$


where

$$
c(q, r, s)= \begin{cases}r^{q / s} & (1 \leq s<\infty, s \neq q) \\ 1 & (1 \leq s<\infty, s=q) \\ 1 & (s=\infty)\end{cases}
$$

Therefore, (8) holds with

$$
\kappa=c(q, r, s), \quad \Phi(x, t)=\sum_{i=1}^{r}\left|p_{i}(x)-p_{i}(t)\right|^{q},
$$

and so taking $p=q$ in Theorems 3.2 and Corollary 3.4, we have the following results which can be more convenient for later applications to the concrete examples of interpolation type operators.

Theorem 5.1. Suppose that $\mathfrak{A}$ is positive and normal. Let $q \geq 1$ and let $\left\{\epsilon_{\alpha}\right\}_{\alpha \in D}$ be a net of positive real numbers. Then the following statements hold:

(a) For all $F \in B C(X, E)$ and all $\alpha \in D$,

$$
E_{\alpha}(F) \leq\left(1+\min \left\{c(q, r, s)^{1 / q} \epsilon_{\alpha}^{-1}, c(q, r, s) \epsilon_{\alpha}^{-q}\right\}\right) \omega\left(F, \epsilon_{\alpha} \zeta_{\alpha}(q)\right),
$$

where

$$
\zeta_{\alpha}(q)=\left(\sup \left\{\sum_{i=1}^{r}\left(\sum_{y \in Y} \chi_{\alpha, \lambda}(x ; y)\left|p_{i}(x)-p_{i}\left(\xi_{\alpha, \lambda}(y)\right)\right|^{q}\right): \lambda \in \Lambda, x \in X_{0}\right\}\right)^{1 / q} .
$$

(b) For all $f \in E_{0}$ and all $\alpha \in D$,

$$
e_{\alpha}(f) \leq\left(1+\min \left\{c(q, r, s)^{1 / q} \epsilon_{\alpha}^{-1}, c(q, r, s) \epsilon_{\alpha}^{-q}\right\}\right) \omega_{\mathfrak{T}}\left(f, \epsilon_{\alpha} \zeta_{\alpha}(q)\right) .
$$

Let $X=[0, \infty)^{r}$ be the region of the first hyperquadrant and let

$$
m_{\alpha, i}: \Lambda \rightarrow \mathbb{N}, \quad a_{\alpha, i}: \Lambda \rightarrow[0, \infty) \quad(\alpha \in D, i=1,2, \ldots, r)
$$

and

$$
I_{\alpha, \lambda}:=\left\{k=\left(k_{1}, k_{2}, \ldots, k_{r}\right) \in \mathbb{N}_{0}^{r}: 0 \leq k_{i} \leq m_{\alpha, i}(\lambda), i=1,2, \ldots, r\right\} .
$$

We define

$$
\chi_{\alpha, \lambda}(x ; k)=\prod_{i=1}^{r}\left(\begin{array}{c}
m_{\alpha, i}(\lambda) \\
k_{i}
\end{array}\right) x_{i}^{k_{i}}\left(1-x_{i}\right)^{m_{\alpha, i}(\lambda)-k_{i}} \quad\left(x \in X, k \in I_{\alpha, \lambda}\right)
$$


and

$$
\xi_{\alpha, \lambda}(k)=\left(a_{\alpha, 1}(\lambda) k_{1}, a_{\alpha, 2}(\lambda) k_{2}, \ldots, a_{\alpha, r}(\lambda) k_{r}\right) \quad\left(k \in I_{\alpha, \lambda}\right) .
$$

Then the interpolation type operators (20) and (21) generalize the $r$-dimensional Bernstein operators, which are defined as follows (cf. [8, 12]):

Let $\mathbb{I}_{r}=[0,1]^{r}$ be the unit $r$-cube and let $\left\{\nu_{n, i}\right\}_{n \in \mathbb{N}}, i=1,2, \ldots, r$, be strictly monotone increasing sequences of positive integers. Then we define

$$
\begin{aligned}
& B_{n}(F)(x)=\sum_{k_{1}=0}^{\nu_{n, 1}} \sum_{k_{2}=0}^{\nu_{n, 2}} \cdots \sum_{k_{r}=0}^{\nu_{n, r}} F\left(\frac{k_{1}}{\nu_{n, 1}}, \frac{k_{2}}{\nu_{n, 2}}, \ldots, \frac{k_{r}}{\nu_{n, r}}\right) \\
& \times \prod_{j=1}^{r}\left(\begin{array}{c}
\nu_{n, j} \\
k_{j}
\end{array}\right) x_{j}^{k_{j}}\left(1-x_{j}\right)^{\nu_{n, j}-k_{j}} \quad\left(F \in C\left(\mathbb{I}_{r}, E\right), x \in \mathbb{I}_{r}\right)
\end{aligned}
$$

and

$$
\begin{aligned}
& C_{n}(x)(f)=\sum_{k_{1}=0}^{\nu_{n, 1}} \sum_{k_{2}=0}^{\nu_{n, 2}} \cdots \sum_{k_{r}=0}^{\nu_{n, r}} T\left(\frac{k_{1}}{\nu_{n, 1}}, \frac{k_{2}}{\nu_{n, 2}}, \ldots, \frac{k_{r}}{\nu_{n, r}}\right)(f) \\
& \times \prod_{j=1}^{r}\left(\begin{array}{c}
\nu_{n, j} \\
k_{j}
\end{array}\right) x_{j}^{k_{j}}\left(1-x_{j}\right)^{\nu_{n, j}-k_{j}} \quad\left(f \in E_{0}, x \in \mathbb{I}_{r}\right) .
\end{aligned}
$$

Now, let $X_{0}$ be a closed subset of $\mathbb{I}_{r}$. Then we have

$$
z_{\alpha}:=\zeta_{\alpha}(2)=\left(\sup \left\{\sum_{i=1}^{r} z_{\alpha, i}(\lambda, x): \lambda \in \Lambda, x \in X_{0}\right\}\right)^{1 / 2},
$$

where

$$
z_{\alpha, i}(\lambda, x)=\left(a_{\alpha, i}(\lambda) m_{\alpha, i}(\lambda)-1\right)^{2} p_{i}^{2}(x)+a_{\alpha, i}^{2}(\lambda) m_{\alpha, i}(\lambda) p_{i}(x)\left(1-p_{i}(x)\right) .
$$

Therefore, applying Theorem 5.1 with $q=2$ we obtain the following estimates:

$$
\begin{aligned}
& E_{\alpha}(F) \leq\left(1+\min \left\{\sqrt{c(r, s)} \epsilon_{\alpha}^{-1}, c(r, s) \epsilon_{\alpha}^{-2}\right\}\right) \omega\left(F, \epsilon_{\alpha} z_{\alpha}\right) \quad(F \in B C(X, E)) \\
& e_{\alpha}(f) \leq\left(1+\min \left\{\sqrt{c(r, s)} \epsilon_{\alpha}^{-1}, c(r, s) \epsilon_{\alpha}^{-2}\right\}\right) \omega_{\mathfrak{T}}\left(f, \epsilon_{\alpha} z_{\alpha}\right) \quad\left(f \in E_{0}\right)
\end{aligned}
$$

Here

$$
c(r, s)= \begin{cases}r^{2 / s} & (1 \leq s<\infty, s \neq 2) \\ 1 & (s=2, \infty) .\end{cases}
$$

In particular, if $m_{\alpha, i}(\lambda) a_{\alpha, i}(\lambda)=1$ for all $\alpha \in D, \lambda \in \Lambda$ and for $i=1,2$, $\ldots, r$, then (22) and (23) hold with

$$
z_{\alpha}=\left(\sup \left\{\sum_{i=1}^{r} \frac{1}{m_{\alpha, i}(\lambda)}\left(p_{i}(x)-p_{i}^{2}(x)\right): \lambda \in \Lambda, x \in X_{0}\right\}\right)^{1 / 2} .
$$


Therefore, for the Bernstein operators, we have

$$
\left\|B_{n}(F)-F\right\|_{X_{0}} \leq\left(1+\min \left\{\sqrt{c(r, s)} \epsilon_{n}^{-1}, c(r, s) \epsilon_{n}^{-2}\right\}\right) \omega\left(F, \epsilon_{n} \theta_{n}\right)
$$

and

$(25)\left\|C_{n}(\cdot)(f)-T(\cdot)(f)\right\|_{X_{0}} \leq\left(1+\min \left\{\sqrt{c(r, s)} \epsilon_{n}^{-1}, c(r, s) \epsilon_{n}^{-2}\right\}\right) \omega_{\mathfrak{T}}\left(f, \epsilon_{n} \theta_{n}\right)$,

where $\left\{\epsilon_{n}\right\}_{n \in \mathbb{N}}$ is a sequence of positive integers and

$$
\theta_{n}:=\left(\max \left\{\sum_{i=1}^{r} \frac{1}{\nu_{n, i}}\left(p_{i}(x)-p_{i}^{2}(x)\right): x \in X_{0}\right\}\right)^{1 / 2} .
$$

In paritucular, (24) and (25) yield the following estimates:

$$
\begin{gathered}
\left\|B_{n}(F)-F\right\|_{\mathbb{I}_{r}} \leq \theta_{n}(r, s) \omega\left(F, \epsilon_{n} \sqrt{\sum_{i=1}^{r} \frac{1}{\nu_{n, i}}}\right) ; \\
\left\|C_{n}(\cdot)(f)-T(\cdot)(f)\right\|_{\mathbb{I}_{r}} \leq \theta_{n}(r, s) \omega_{\mathfrak{T}}\left(f, \epsilon_{n} \sqrt{\sum_{i=1}^{r} \frac{1}{\nu_{n, i}}}\right) .
\end{gathered}
$$

Here

$$
\theta_{n}(r, s):=1+\min \left\{\frac{\sqrt{c(r, s)}}{2 \epsilon_{n}}, \frac{c(r, s)}{4 \epsilon_{n}^{2}}\right\} .
$$

Let $\left\{T_{i}(t): t \in[0,1], i=1,2, \ldots, r\right\}$ be a family of strongly continuous mappings from $E_{0}$ to itself such that for every $t, u \in[0,1], t T_{i}(u)$ commutes with $(1-t) I$, where $I$ is the identity operator on $E$ and $T_{i}(v)^{n}=T_{i}(n v)$ whenever $v \in$ $[0,1], n \in \mathbb{N}_{0}$ and $n v \in[0,1]$. If $T(x)=\prod_{i=1}^{r} T_{i}\left(x_{i}\right)$ for all $x=\left(x_{1}, x_{2}, \ldots, x_{r}\right) \in$ $\mathbb{I}_{r}$, then

$$
C_{n}(x)(f)=\prod_{i=1}^{r}\left(\left(1-x_{i}\right) I+x_{i} T_{i}\left(\frac{1}{\nu_{n, i}}\right)\right)^{\nu_{n, i}}(f)=\prod_{i=1}^{r}\left(I+x_{i}\left(T_{i}\left(\frac{1}{\nu_{n, i}}\right)-I\right)\right)^{\nu_{n, i}}(f) .
$$

Therefore, the inequality (27) estimates the rate of convergence in [11, Theorem 5] for $r=1$, which improves the estimate in [3, Proposition 1.2.9] and furthermore, it generalizes and improves the convergence rate in [4, Theorem 1.1]. Also, we note that in view of Lemma 2.6, other results of [4] can be generalized and improved by the same argument as the above manner.

Suppose that $\mathcal{A}$ is stochastic, and let $\left\{\ell_{n}\right\}_{n \in \mathbb{N}_{0}}$ be a sequence of positive integers. We define

$$
K_{\alpha, \lambda}(F)(x)=\sum_{n=0}^{\infty} a_{\alpha, n}^{(\lambda)} B_{\ell_{n}}(F)(x) \quad\left(F \in C\left(\mathbb{I}_{r}, E\right), x \in \mathbb{I}_{r}\right)
$$


and

$$
L_{\alpha, \lambda}(x)(f)=\sum_{n=0}^{\infty} a_{\alpha, n}^{(\lambda)} C_{\ell_{n}}(x)(f) \quad\left(f \in E_{0}, x \in \mathbb{I}_{r}\right) .
$$

Then we have the following estimates:

$$
\begin{aligned}
& E_{\alpha}(F) \leq\left(1+\min \left\{\sqrt{c(r, s)} \epsilon_{\alpha}^{-1}, c(r, s) \epsilon_{\alpha}^{-2}\right\}\right) \omega\left(F, \epsilon_{\alpha} \eta_{\alpha}\right) \\
& e_{\alpha}(f) \leq\left(1+\min \left\{\sqrt{c(r, s)} \epsilon_{\alpha}^{-1}, c(r, s) \epsilon_{\alpha}^{-2}\right\}\right) \omega_{\mathfrak{T}}\left(f, \epsilon_{\alpha} \eta_{\alpha}\right) .
\end{aligned}
$$

Here

$$
\eta_{\alpha}:=\left(\sup \left\{\sum_{n=0}^{\infty} a_{\alpha, n}^{(\lambda)} \theta_{\ell_{n}}^{2}: \lambda \in \Lambda\right\}\right)^{1 / 2} .
$$

Indeed, we have

$$
\nu_{\alpha}^{2}(2) \leq \sup \left\{\sum_{n=0}^{\infty} a_{\alpha, n}^{(\lambda)}\left(c(r, s) \sum_{i=1}^{r} \frac{p_{i}(x)-p_{i}^{2}(x)}{\nu_{\ell_{n}, i}}\right): \lambda \in \Lambda, x \in X_{0}\right\}=c(r, s) \eta_{\alpha}^{2},
$$

and so, by putting $c(r, s)^{-1 / 2} \epsilon_{\alpha}$ instead of $\epsilon_{\alpha}$, (28) follows from Lemma 2.4 (b) and Theorem 4.1.

The statements analogous to the above results hold for the following settings: Let $X_{0}$ be a closed subset of $\Delta_{r}$, where

$$
\Delta_{r}:=\left\{x=\left(x_{1}, x_{2}, \ldots, x_{r}\right) \in \mathbb{R}^{r}: x_{i} \geq 0, i=1,2, \ldots, r, \sum_{i=1}^{r} x_{i} \leq 1\right\}
$$

is the standard $r$-simplex;

$$
\begin{aligned}
I_{\alpha, \lambda} & :=\left\{k=\left(k_{1}, k_{2}, \ldots, k_{r}\right) \in \mathbb{N}_{0}^{r}: k_{1}+k_{2}+\cdots+k_{r} \leq m_{\alpha}(\lambda)\right\} ; \\
\chi_{\alpha, \lambda}(x ; k) & :=\left(\begin{array}{c}
m_{\alpha}(\lambda) \\
k
\end{array}\right) \prod_{i=1}^{r} x_{i}^{k_{i}}\left(1-\sum_{j=1}^{r} x_{j}\right)^{m_{\alpha}(\lambda)-\Sigma_{j=1}^{r} k_{j}} \quad\left(x \in X, k \in I_{\alpha, \lambda}\right),
\end{aligned}
$$

where $m_{\alpha}: \Lambda \rightarrow \mathbb{N}$, and

$$
\begin{gathered}
\left(\begin{array}{c}
m_{\alpha}(\lambda) \\
k
\end{array}\right):=\frac{m_{\alpha}(\lambda) !}{k_{1} ! k_{2} ! \cdots k_{r} !\left(m_{\alpha}(\lambda)-k_{1}-k_{2}-\cdots-k_{r}\right) !} \\
\xi_{\alpha, \lambda}(k):=\left(a_{\alpha, 1}(\lambda) k_{1}, a_{\alpha, 2}(\lambda) k_{2}, \ldots, a_{\alpha, r}(\lambda) k_{r}\right) \quad\left(k \in I_{\alpha, \lambda}\right) .
\end{gathered}
$$

Next, let $X=[-1,1]^{r}$ and let $X_{0}$ be a closed subset of $X$. Let $Q_{n}(t)=$ $\cos (n \arccos t)$ be the Chebyshev polynomial of degree $n$, and let $t_{n, j}, j=1,2, \ldots, n$, be zeros of $Q_{n}(t)$, i.e.,

$$
t_{n, j}=\cos \left(\frac{2 j-1}{2 n} \pi\right), \quad(j=1,2, \ldots, n) .
$$


Let

$$
m_{\alpha, i}: \Lambda \rightarrow \mathbb{N}, \quad a_{\alpha, i}: \Lambda \rightarrow[-1,1] \quad(\alpha \in D, i=1,2, \ldots, r)
$$

and let

$$
N_{\alpha, \lambda}:=\left\{k=\left(k_{1}, k_{2}, \ldots, k_{r}\right) \in \mathbb{N}^{r}: 1 \leq k_{i} \leq m_{\alpha, i}(\lambda), i=1,2, \ldots, r\right\} .
$$

We define

$$
\chi_{\alpha, \lambda}(x ; k)=\prod_{i=1}^{r} \chi_{m_{\alpha, i}(\lambda)}\left(x_{i} ; k_{i}\right) \quad\left(x \in X, k \in N_{\alpha, \lambda}\right),
$$

where

$$
\chi_{m_{\alpha, i}(\lambda)}\left(x_{i} ; k_{i}\right)=\left(1-x_{i} t_{m_{\alpha, i}(\lambda), k_{i}}\right)\left\{\frac{Q_{m_{\alpha, i}(\lambda)}\left(x_{i}\right)}{m_{\alpha, i}(\lambda)\left(x_{i}-t_{m_{\alpha, i}(\lambda), k_{i}}\right)}\right\}^{2}
$$

and

$$
\xi_{\alpha, \lambda}(k)=\left(a_{\alpha, 1}(\lambda) t_{m_{\alpha, 1}(\lambda), k_{1}}, \ldots, a_{\alpha, r}(\lambda) t_{m_{\alpha, r}(\lambda), k_{r}}\right) \quad\left(k \in N_{\alpha, \lambda}\right) .
$$

Then the interpolation type operators (20) and (21) generalize the $r$-dimensinal Hermite-Fejer operators, which are defined as follows:

We define

$$
\begin{gathered}
H_{n}(F)(x)=\sum_{k_{1}=1}^{\nu_{n, 1}} \sum_{k_{2}=1}^{\nu_{n, 2}} \cdots \sum_{k_{r}=1}^{\nu_{n, r}} F\left(t_{\nu_{n, 1}, k_{1}}, t_{\nu_{n, 2}, k_{2}}, \ldots, t_{\nu_{n, r}, k_{r}}\right) \\
\times \prod_{j=1}^{r}\left(1-x_{j} t_{\nu_{n, j}, k_{j}}\right)\left\{\frac{Q_{\nu_{n, j}}\left(x_{j}\right)}{\nu_{n, j}\left(x_{j}-t_{\nu_{n, j}, k_{j}}\right)}\right\}^{2} \quad(F \in C(X, E), x \in X)
\end{gathered}
$$

and

$$
\begin{aligned}
& G_{n}(x)(f)=\sum_{k_{1}=1}^{\nu_{n, 1}} \sum_{k_{2}=1}^{\nu_{n, 2}} \cdots \sum_{k_{r}=1}^{\nu_{n, r}} T\left(t_{\nu_{n, 1}, k_{1}}, t_{\nu_{n, 2}, k_{2}}, \ldots, t_{\nu_{n, r}, k_{r}}\right)(f) \\
& \times \prod_{j=1}^{r}\left(1-x_{j} t_{\nu_{n, j}, k_{j}}\right)\left\{\frac{Q_{\nu_{n, j}}\left(x_{j}\right)}{\nu_{n, j}\left(x_{j}-t_{\nu_{n, j}, k_{j}}\right)}\right\}^{2} \quad\left(f \in E_{0}, x \in X\right) .
\end{aligned}
$$

Now, we have

$$
y_{\alpha}:=\zeta_{\alpha}(2)=\left(\sup \left\{\sum_{i=1}^{r} y_{\alpha, i}(\lambda, x): \lambda \in \Lambda, x \in X_{0}\right\}\right)^{1 / 2}
$$


where

$$
\begin{aligned}
y_{\alpha, i}(\lambda, x)= & \frac{Q_{m_{\alpha, i}(\lambda)}^{2}\left(x_{i}\right)}{m_{\alpha, i}(\lambda)}-2 x_{i}\left(a_{\alpha, i}(\lambda)-1\right) \sum_{k_{i}=1}^{m_{\alpha, i}(\lambda)} t_{m_{\alpha, i}(\lambda), k_{i}} \chi_{m_{\alpha, i}(\lambda)}\left(x_{i} ; k_{i}\right) \\
& +\left(a_{\alpha, i}^{2}(\lambda)-1\right) \sum_{k_{i}=1}^{m_{\alpha, i}(\lambda)} t_{m_{\alpha, i}(\lambda), k_{i}}{ }^{2} \chi_{m_{\alpha, i}(\lambda)}\left(x_{i} ; k_{i}\right) .
\end{aligned}
$$

Therefore, applying Theorem 5.1 with $q=2$ we get the following estimates:

$$
\begin{aligned}
& E_{\alpha}(F) \leq\left(1+\min \left\{\sqrt{c(r, s)} \epsilon_{\alpha}^{-1}, c(r, s) \epsilon_{\alpha}^{-2}\right\}\right) \omega\left(F, \epsilon_{\alpha} y_{\alpha}\right) ; \\
& e_{\alpha}(f) \leq\left(1+\min \left\{\sqrt{c(r, s)} \epsilon_{\alpha}^{-1}, c(r, s) \epsilon_{\alpha}^{-2}\right\}\right) \omega_{\mathfrak{T}}\left(f, \epsilon_{\alpha} y_{\alpha}\right) .
\end{aligned}
$$

In particular, if $a_{\alpha, i}(\lambda)=1$ for all $\alpha \in D, \lambda \in \Lambda$ and for $i=1,2, \ldots, r$, then (29) and (30) hold with

$$
y_{\alpha}=\left(\sup \left\{\sum_{i=1}^{r} \frac{\left(Q_{m_{\alpha, i}(\lambda)} \circ p_{i}\right)^{2}(x)}{m_{\alpha, i}(\lambda)}: \lambda \in \Lambda, x \in X_{0}\right\}\right)^{1 / 2} .
$$

Therefore, for the Hermite-Fejér operators, (29) and (30) yield

$$
\left\|H_{n}(F)-F\right\|_{X_{0}} \leq\left(1+\min \left\{\sqrt{c(r, s)} \epsilon_{n}^{-1}, c(r, s) \epsilon_{n}^{-2}\right\}\right) \omega\left(F, \epsilon_{n} \tau_{n}\right)
$$

and

$$
\left\|G_{n}(\cdot)(f)-T(\cdot)(f)\right\|_{X_{0}} \leq\left(1+\min \left\{\sqrt{c(r, s)} \epsilon_{n}^{-1}, c(r, s) \epsilon_{n}^{-2}\right\}\right) \omega_{\mathfrak{T}}\left(f, \epsilon_{n} \tau_{n}\right),
$$

respectively, where

$$
\tau_{n}=\left(\max \left\{\sum_{i=1}^{r} \frac{\left(Q_{\nu_{n, i}} \circ p_{i}\right)^{2}(x)}{\nu_{n, i}}: x \in X_{0}\right\}\right)^{1 / 2} .
$$

In particular, the following estimates hold:

$$
\begin{gathered}
\left\|H_{n}(F)-F\right\|_{X} \leq\left(1+\min \left\{\sqrt{c(r, s)} \epsilon_{n}^{-1}, c(r, s) \epsilon_{n}^{-2}\right\}\right) \omega\left(F, \epsilon_{n} \sqrt{\sum_{i=1}^{r} \frac{1}{\nu_{n, i}}}\right) \\
\left\|G_{n}(\cdot)(f)-T(\cdot)(f)\right\|_{X} \leq\left(1+\min \left\{\sqrt{c(r, s)} \epsilon_{n}^{-1}, c(r, s) \epsilon_{n}^{-2}\right\}\right) \omega_{\mathfrak{T}}\left(f, \epsilon_{n} \sqrt{\sum_{i=1}^{r} \frac{1}{\nu_{n, i}}}\right) .
\end{gathered}
$$


Suppose again that $\mathcal{A}$ is stochastic, and we define

$$
K_{\alpha, \lambda}(F)(x)=\sum_{n=0}^{\infty} a_{\alpha, n}^{(\lambda)} H_{\ell_{n}}(F)(x) \quad(F \in C(X, E), x \in X)
$$

and

$$
L_{\alpha, \lambda}(x)(f)=\sum_{n=0}^{\infty} a_{\alpha, n}^{(\lambda)} G_{\ell_{n}}(x)(f) \quad\left(f \in E_{0}, x \in X\right) .
$$

Then we have the following estimates:

$$
\begin{aligned}
& E_{\alpha}(F) \leq\left(1+\min \left\{\sqrt{c(r, s)} \epsilon_{\alpha}^{-1}, c(r, s) \epsilon_{\alpha}^{-2}\right\}\right) \omega\left(F, \epsilon_{\alpha} \delta_{\alpha}\right) \\
& e_{\alpha}(f) \leq\left(1+\min \left\{\sqrt{c(r, s)} \epsilon_{\alpha}^{-1}, c(r, s) \epsilon_{\alpha}^{-2}\right\}\right) \omega_{\mathfrak{T}}\left(f, \epsilon_{\alpha} \delta_{\alpha}\right) .
\end{aligned}
$$

Here

$$
\delta_{\alpha}=\left(\sup \left\{\sum_{n=0}^{\infty} a_{\alpha, n}^{(\lambda)} \tau_{\ell_{n}}^{2}: \lambda \in \Lambda\right\}\right)^{1 / 2}
$$

Indeed, we have

$$
\nu_{\alpha}^{2}(2) \leq \sup \left\{\sum_{n=0}^{\infty} a_{\alpha, n}^{(\lambda)}\left(c(r, s) \sum_{i=1}^{r} \frac{\left(Q_{\nu_{\ell_{n}, i}} \circ p_{i}\right)^{2}(x)}{\nu_{\ell_{n}, i}}\right): \lambda \in \Lambda, x \in X_{0}\right\}=c(r, s) \delta_{\alpha}^{2}
$$

and so, by putting $c(r, s)^{-1 / 2} \epsilon_{\alpha}$ instead of $\epsilon_{\alpha}$, (31) follows from Lemma 2.4 (b) and Theorem 4.1.

\section{Convolution Type Operators}

In this section, we treat the equi-uniform $\mathcal{A}$-summation processes of convolution type operators defined by (13) and (14) with (15) and (16), where $X=Y$ is a convex subset of $\mathbb{R}^{r}$ with the metric $d=d_{s}, 1 \leq s \leq \infty$, defined in Section 5 and $\xi_{n}(y)=y$ for all $n \in \mathbb{N}_{0}$ and all $y \in X$. Here we suppose that $\mathcal{A}$ is stochastic.

Let $c>0$ and let $\left\{g_{n}\right\}_{n \in \mathbb{N}_{0}}$ be a sequence of nonnegative even continuous functions on $[-c, c]$ such that $\int_{-c}^{c} g_{n}(t) d t=1$ for all $n \in \mathbb{N}_{0}$. Let

$$
X=\prod_{i=1}^{r}\left[a_{i}, b_{i}\right], \quad 0<b_{i}-a_{i} \leq c, \quad i=1,2, \ldots, r
$$

and

$$
X_{0}=\prod_{i=1}^{r}\left[a_{i}+\delta_{i}, b_{i}-\delta_{i}\right], \quad 0<\delta_{i}<\frac{1}{2}\left(b_{i}-a_{i}\right), \quad i=1,2, \ldots, r .
$$


We define

$$
\chi_{n}(x ; y)=\prod_{i=1}^{r}\left(g_{n} \circ p_{i}\right)(x-y) \quad\left(x, y \in X, n \in \mathbb{N}_{0}\right) .
$$

Let $\left\{\epsilon_{\alpha}\right\}_{\alpha \in D}$ be a net of positive real numbers.

\section{Theorem 6.1.}

(a) For all $F \in C(X, E)$ and all $\alpha \in D$,

$$
E_{\alpha}(F) \leq\|F\|_{X_{0}} \zeta_{\alpha}^{2} \sum_{i=1}^{r} \frac{1}{\delta_{i}^{2}}+\left(1+\min \left\{\sqrt{r c(r, s)} \epsilon_{\alpha}^{-1}, r c(r, s) \epsilon_{\alpha}^{-2}\right\}\right) \omega\left(F, \epsilon_{\alpha} \zeta_{\alpha}\right),
$$

where

$$
\zeta_{\alpha}=\left(\sup \left\{\sum_{m=0}^{\infty} a_{\alpha, m}^{(\lambda)} \int_{-c}^{c} t^{2} g_{m}(t) d t: \lambda \in \Lambda\right\}\right)^{1 / 2}
$$

(b) For all $f \in E_{0}$ and all $\alpha \in D$,

$$
\begin{aligned}
e_{\alpha}(f) & \leq\|T(\cdot)(f)\|_{X_{0}} \zeta_{\alpha}^{2} \sum_{i=1}^{r} \frac{1}{\delta_{i}^{2}} \\
& +\left(1+\min \left\{\sqrt{r c(r, s)} \epsilon_{\alpha}^{-1}, r c(r, s) \epsilon_{\alpha}^{-2}\right\}\right) \omega_{\mathfrak{T}}\left(f, \epsilon_{\alpha} \zeta_{\alpha}\right) .
\end{aligned}
$$

Proof. By [11, Lemma 4], we have

$$
\begin{gathered}
\int_{X} \chi_{n}(x ; y) d y \leq 1 \quad\left(n \in \mathbb{N}_{0}, x \in X\right), \\
0 \leq 1-\int_{X} \chi_{n}(x ; y) \leq\left(\int_{-c}^{c} t^{2} g_{n}(t) d t\right) \sum_{i=1}^{r} \frac{1}{\delta_{i}^{2}} \quad\left(n \in \mathbb{N}_{0}, x \in X_{0}\right)
\end{gathered}
$$

and

$$
\int_{X} \chi_{n}(x ; y) d_{2}^{2}(x, y) d y \leq r \int_{-c}^{c} t^{2} g_{n}(t) d t \quad\left(n \in \mathbb{N}_{0}, x \in X\right) .
$$

Therefore, we obtain $b_{\alpha, \lambda}(x) \leq 1$,

$$
\tau_{\alpha}=\sup \left\{\sum_{m=0}^{\infty} a_{\alpha, m}^{(\lambda)}\left(1-\int_{X} \chi_{m}(x ; y) d y\right): \lambda \in \Lambda, x \in X_{0}\right\} \leq \zeta_{\alpha}^{2} \sum_{i=1}^{r} \frac{1}{\delta_{i}^{2}}
$$

and

$$
\nu_{\alpha}^{2}(2)=\sup \left\{\sum_{m=0}^{\infty} a_{\alpha, m}^{(\lambda)} \int_{X} \chi_{m}(x ; y) d_{s}^{2}(x, y) d y: \lambda \in \Lambda, x \in X_{0}\right\} \leq r c(r, s) \zeta_{\alpha}^{2} .
$$


Thus, by selecting $p=2$ and by putting $(r c(r, s))^{-1 / 2} \epsilon_{\alpha}$ instead of $\epsilon_{\alpha}$, the desired result follows from Theorem 4.1 and Lemma 2.4 (b).

Let $\varphi$ be a nonnegative, continuous even function on $[-c, c]$ such that $\varphi$ is decreasing on $[0, c]$ and

$$
\varphi(0)=1, \quad 0 \leq \varphi(t)<1 \quad(0<t \leq c) .
$$

We define

$$
g_{n}(t)=\rho_{n} \varphi^{n}(t) \quad(|t| \leq c), \quad \text { where } \quad \rho_{n}=\left(\int_{-c}^{c} \varphi^{n}(t) d t\right)^{-1} \quad\left(n \in \mathbb{N}_{0}\right) .
$$

Then we have

$$
\chi_{n}(x ; y)=\rho_{n}^{r} \prod_{i=1}^{r}\left(\varphi^{n} \circ p_{i}\right)(x-y) \quad(x, y \in X),
$$

which reduce to the Korovkin kernels in case $r=1$ (cf. [6]).

\section{Corollary 6.2. Suppose that}

$$
\lim _{t \rightarrow+0} \frac{1-\varphi(t)}{t^{p}}=q
$$

for some $p>0$ and $q>0$. Then the following statements hold:

(a) For all $F \in C(X, E)$ and all $\alpha \in D$,

$$
E_{\alpha}(F) \leq C_{\varphi}\|F\|_{X_{0}} \eta_{\alpha}^{2} \sum_{i=1}^{r} \frac{1}{\delta_{i}^{2}}+\left(1+\min \left\{\sqrt{r c(r, s) C_{\varphi}}, r c(r, s) C_{\varphi}\right\}\right) \omega\left(F, \eta_{\alpha}\right),
$$

where

$$
C_{\varphi}=\sup \left\{(n+1)^{2 / p} \int_{-c}^{c} t^{2} g_{n}(t) d t: n \in \mathbb{N}_{0}\right\}
$$

and

$$
\eta_{\alpha}=\left(\sup \left\{\sum_{m=0}^{\infty} \frac{a_{\alpha, m}^{(\lambda)}}{(m+1)^{2 / p}}: \lambda \in \Lambda\right\}\right)^{1 / 2}
$$

(b) For all $f \in E_{0}$ and all $\alpha \in D$,

$$
\begin{aligned}
e_{\alpha}(f) & \leq C_{\varphi}\|T(\cdot)(f)\|_{X_{0}} \eta_{\alpha}^{2} \sum_{i=1}^{r} \frac{1}{\delta_{i}^{2}} \\
& +\left(1+\min \left\{\sqrt{r c(r, s) C_{\varphi}}, r c(r, s) C_{\varphi}\right\}\right) \omega_{\mathfrak{T}}\left(f, \eta_{\alpha}\right) .
\end{aligned}
$$


Indeed, from the proof of [2, Theorem 1] (cf. [2, Lemma 2]), we see that $C_{\varphi}$ is finite. Therefore, we have $\zeta_{\alpha} \leq \sqrt{C_{\varphi}} \eta_{\alpha}$ for all $\alpha \in D$. Thus, by selecting $\epsilon_{\alpha}=C_{\varphi}^{-1 / 2}$, the desired result follows from Theorem 6.1 .

For several important examples of $\varphi$ satisfying Condition (32), see [2] and [12].

Next, we consider the convolution type operators for the whole space $\mathbb{R}^{r}$. Let $\left\{h_{n}\right\}_{n \in \mathbb{N}_{0}}$ be a sequence of nonnegative Lebesgue integrable functions on $\mathbb{R}$ such that $\int_{\mathbb{R}} h_{n}(t) d t=1$ for all $n \in \mathbb{N}_{0}$. Let $X=\mathbb{R}^{r}$ and we define

$$
\chi_{n}(x ; y)=\prod_{i=1}^{r}\left(h_{n} \circ p_{i}\right)(x-y) \quad\left(x, y \in X, n \in \mathbb{N}_{0}\right) .
$$

Theorem 6.3. Let $q \geq 1$.

(a) For all $F \in B C(X, E)$ and all $\alpha \in D$,

$$
E_{\alpha}(F) \leq\left(1+\min \left\{(r c(q, r, s))^{1 / q} \epsilon_{\alpha}^{-1}, r c(q, r, s) \epsilon_{\alpha}^{-q}\right\}\right) \omega\left(F, \epsilon_{\alpha} \theta_{\alpha}(q)\right),
$$

where

$$
\theta_{\alpha}(q)=\left(\sup \left\{\sum_{m=0}^{\infty} a_{\alpha, m}^{(\lambda)} \int_{\mathbb{R}}|t|^{q} h_{m}(t) d t: \lambda \in \Lambda\right\}\right)^{1 / q}<\infty .
$$

(b) For all $f \in E_{0}$ and all $\alpha \in D$,

$$
e_{\alpha}(f) \leq\left(1+\min \left\{\left(r(c(q, r, s))^{1 / q} \epsilon_{\alpha}^{-1}, r c(q, r, s) \epsilon_{\alpha}^{-q}\right\}\right) \omega_{\mathfrak{T}}\left(f, \epsilon_{\alpha} \theta_{\alpha}(q)\right) .\right.
$$

Proof. We have

$$
\int_{X} \chi_{n}(x ; y) d y=1 \quad\left(n \in \mathbb{N}_{0}, x \in X\right),
$$

and so $\tau_{\alpha}=0$ and $b_{\alpha, \lambda}(x)=1$. Also, we have

$$
\begin{gathered}
\sum_{m=0}^{\infty} a_{\alpha, m}^{(\lambda)} \int_{X} \chi_{m}(x ; y) d^{q}(x, y) d y \leq c(q, r, s) \sum_{m=0}^{\infty} a_{\alpha, m}^{(\lambda)} \sum_{i=1}^{r} \int_{\mathbb{R}} h_{m}\left(x_{i}-y_{i}\right)\left|x_{i}-y_{i}\right|^{q} d y_{i} \\
=r c(q, r, s) \sum_{m=0}^{\infty} a_{\alpha, m}^{(\lambda)} \int_{\mathbb{R}}|t|^{q} h_{m}(t) d t
\end{gathered}
$$

and so $\nu_{\alpha}^{q}(q) \leq r c(q, r, s) \theta_{\alpha}^{q}(q)$. Thus, by taking $p=q$ and by putting $(r c(q, r, s))^{-1 / q_{\epsilon}} \epsilon_{\alpha}$ instead of $\epsilon_{\alpha}$, the desired result follows from Lemma 2.4 (b) and Theorem 4.1. 
Let $\left\{k_{n}\right\}_{n \in \mathbb{N}_{0}}$ be a sequence of nonnegatve, even $2 \pi$-periodic, Lebesgue integrable functions on $\mathbb{R}$ having Fourier series expansions

$$
k_{n}(t) \sim \sum_{j=-\infty}^{\infty} \hat{k_{n}}(j) e^{i j t}, \quad \hat{k_{n}}(j):=\frac{1}{2 \pi} \int_{-\pi}^{\pi} k_{n}(t) e^{-i j t} d t
$$

with $\hat{k_{n}}(0)=1$, and we define

$$
h_{n}(t)= \begin{cases}\frac{1}{2 \pi} k_{n}(t) & (|t| \leq \pi) \\ 0 & (|t|>\pi) .\end{cases}
$$

\section{Corollary 6.4.}

(a) For all $F \in B C(X, E)$ and all $\alpha \in D$,

$$
E_{\alpha}(F) \leq\left(1+\min \left\{\pi \sqrt{\frac{r c(r, s)}{2}} \epsilon_{\alpha}^{-1}, \frac{\pi^{2} r c(r, s)}{2} \epsilon_{\alpha}^{-2}\right\}\right) \omega\left(F, \epsilon_{\alpha} \eta_{\alpha}\right),
$$

where

$$
\eta_{\alpha}=\left(\sup \left\{\sum_{m=0}^{\infty} a_{\alpha, m}^{(\lambda)}\left(1-\hat{k_{m}}(1)\right): \lambda \in \Lambda\right\}\right)^{1 / 2} .
$$

(b) For all $f \in E_{0}$ and all $\alpha \in D$,

$$
e_{\alpha}(f) \leq\left(1+\min \left\{\pi \sqrt{\frac{r c(r, s)}{2}} \epsilon_{\alpha}^{-1}, \frac{\pi^{2} r c(r, s)}{2} \epsilon_{\alpha}^{-2}\right\}\right) \omega_{\mathfrak{T}}\left(f, \epsilon_{\alpha} \eta_{\alpha}\right) .
$$

Indeed, by the inequality $(2 / \pi) t \leq \sin t(0 \leq t \leq \pi / 2)$, we have

$$
\int_{-\pi}^{\pi} t^{2} k_{m}(t) d t \leq \pi^{2} \int_{-\pi}^{\pi} k_{m}(t) \sin ^{2} \frac{t}{2} d t=\frac{\pi^{2}}{2} \int_{-\pi}^{\pi}(1-\cos t) k_{m}(t) d t \quad\left(m \in \mathbb{N}_{0}\right) .
$$

Thus, we have $\theta_{\alpha}(2) \leq(\pi / \sqrt{2}) \eta_{\alpha}$, and so putting $(\pi / \sqrt{2})^{-1} \epsilon_{\alpha}$ instead of $\epsilon_{\alpha}$ the desired result follows from Theorem 6.3.

Note that by the same argument as the above proof, Theorem 6.1 is applied for $c=\pi$ and $g_{n}=(1 / 2 \pi) k_{n}$, since $\zeta_{\alpha} \leq(\pi / \sqrt{2}) \eta_{\alpha}$.

Let $\left(\lambda_{n}(j)\right)(n, j=1,2, \ldots)$ be a lower triangular infinite matrix of real numbers and we define

$$
k_{0}(t)=1, \quad k_{n}(t)=1+2 \sum_{j=1}^{n} \lambda_{n}(j) \cos j t \quad(n \in \mathbb{N}, t \in \mathbb{R}) .
$$


Then applying Abel's transformation twice to the function $k_{n}(t)$, we have

$$
k_{n}(t)=\sum_{j=0}^{n-1}(j+1) F_{j}(t) \Delta^{2} \lambda_{n}(j)+(n+1) \lambda_{n}(n) F_{n}(t), \quad \lambda_{n}(0)=1,
$$

where $F_{m}(t)$ is the $m$ th Fejer kernel and

$$
\Delta^{2} \lambda_{n}(j)=\lambda_{n}(j)-2 \lambda_{n}(j+1)+\lambda_{n}(j+2) .
$$

Therefore, if $\lambda_{n}(n) \geq 0$ and $\left\{\lambda_{n}(j)\right\}_{j \in \mathbb{N}_{0}}$ is convex, i.e., $\Delta^{2} \lambda_{n}(j) \geq 0$ for all $j \in \mathbb{N}_{0}$, then $k_{n}(t)$ is a nonnegative, even trigonometric polynomial of degree at most $n$ and so Corollary 6.4 (a) and (b) hold with

$$
\eta_{\alpha}=\left(\sup \left\{\sum_{m=0}^{\infty} a_{\alpha, m}^{(\lambda)}\left(1-\lambda_{m}(1)\right): \lambda \in \Lambda\right\}\right)^{1 / 2} .
$$

For several examples of $\lambda_{n}(j)$ which produce important positive summability kernels, see [12].

Moreover, there is a wide variety of examples of nonperiodic functions $h_{n}(t)$ for which Theorem 6.3 can be applied, from a probabilistic point of view. These can be induced by various probability density functions (see [12]).

\section{REFERENCES}

1. H. T. Bell, Order summability and almost convergence, Proc. Amer. Math. Soc. 38 (1973), 548-552.

2. R. Bojanic and O. Shisha, On the precision of uniform approximation of continuous functions by certain linear positive operators of convolution type, J. Approx. Theory 8 (1973), 101-113.

3. P. L. Butzer and H. Berens, Semi-Groups of Operators and Approximation, SpringerVerlag, Berlin/Heidelberg/New York, 1967.

4. P.-S. Huang and S.-Y. Shaw, Convergence rates of representation formulas for mparameter semigroups, Vietnam J. Math. 30 (2002), 487-500.

5. J. P. King and J. J. Swetits, Positive linear operators and summability, J. Austral. Math. Soc. 11 (1970), 281-290.

6. P. P. Korovkin, Linear Operators and Approximation Theory, Hindustan Publ. Corp., Delhi, 1960.

7. G. G. Lorentz, A contribution to the theory of divergent sequences, Acta Math. 80 (1948), 167-190.

8. G. G. Lorentz, Bernstein Polynomials, Univ. of Toronto Press, Toronto, 1953. 
9. T. Nishishiraho, Saturation of multiplier operators in Banach spaces, Tôhoku Math. J. 34 (1982), 23-42.

10. T. Nishishiraho, Convergence of positive linear approximation processes, Tohoku Math. J. 35 (1983), 441-458.

11. T. Nishishiraho, Approximation processes of integral operators in Banach spaces, $J$. Nonlinear and Convex Analysis 4 (2003), 125-140.

12. T. Nishishiraho, The convergence of equi-uniform approximation processes of integral operators in Banach spaces, Ryukyu Math. J. 16 (2003), 79-111.

13. T. Nishishiraho, The degree of convergence of equi-uniform approximation processes of integral operators in Banach spaces, in Proc. the 3rd Internat. Conf. on Nonlinear Analysis and Convex Analysis, to appear.

14. G. M. Petersen, Almost convergence and uniformly distributed sequences, Quart. J. Math. 7 (1956), 188-191.

15. S.-Y. Shaw, Approximation of unbounded functions and applications to representations of semigroups, J. Approx. Theory 28 (1980), 238-259.

Toshihiko Nishishiraho

Department of Mathematical Sciences,

Faculty of Science,

University of the Ryukyus,

Nishihara-cho, Okinawa 903-0213,

Japan

E-mail: nisiraho@sci.u-ryukyu.ac.jp 